The title for this Special Section is The Developing Brain: Evidence for Plasticity during Childhood and Adolescence, edited by Amanda E. Guyer, Koraly PérezEdgar, and Eveline A. Crone

\title{
Opportunities for Neurodevelopmental Plasticity From Infancy Through Early Adulthood
}

\author{
Amanda E. Guyer \\ University of California, Davis
}

\author{
Koraly Pérez-Edgar \\ The Pennsylvania State University
}

\author{
Eveline A. Crone \\ Leiden University
}

\begin{abstract}
Multiple and rapid changes in brain development occur in infancy and early childhood that undergird behavioral development in core domains. The period of adolescence also carries a second influx of growth and change in the brain to support the unique developmental tasks of adolescence. This special section documents two core conclusions from multiple studies. First, evidence for change in brain-based metrics that underlie cognitive and behavioral functions are not limited to narrow windows in development, but are evident from infancy into early adulthood. Second, the specific evident changes are unique to challenges and goals that are salient for a respective developmental period. These brain-based changes interface with environmental inputs, whether from the child's broader ecology or at an individual level.
\end{abstract}

Brain development is an extraordinary process full of both rapid and gradual transitions designed to establish efficient structural and functional neural connections supporting our behaviors, cognitions, and emotions. The field of developmental neuroscience has burgeoned over the last 20 years with advances in technology and methods that are wellsuited for measuring the human brain in vivo in infants, children, and adolescents. We can now more than ever before directly capture the interplay of changes in experience and brain development

This work was supported by grants from the National Institutes of Health (R01 MH093605 [Amanda E. Guyer], R01 MH098370 [Amanda E. Guyer], R01 MH109692 [Koraly PérezEdgar]), and the University of California (UC) Consortium on the Developmental Science of Adolescence awarded from the UC Office of the President, Multicampus Research Programs and Initiatives (Grant ID: MRP-17-454825) to Amanda E. Guyer. This work was also supported by the Netherlands Organization for Scientific Research (NWO-VICI 453-14-001) and an innovative ideas grant of the European Research Council (ERC CoG PROSOCIAL 681632) to Eveline A. Crone.

Correspondence concerning this article should be addressed to Amanda E. Guyer, Department of Human Ecology, Center for Mind and Brain, University of California, Davis, 267 Cousteau Place, Davis, CA 95618. Electronic mail may be sent to aeguyer@ucdavis.edu. across the life span. As a result, these advances have afforded us a better understanding of when, where, how, and for whom, the brain's structure and function changes over time in response to, and in anticipation of, experience.

Two guiding principles in developmental neuroscience suggest that (a) multiple and rapid brain changes in infancy and early childhood foster development in core domains of functioning, and (b) a second epoch of growth and change in the brain occurs from adolescence into early adulthood, supporting new areas of functioning related to the unique tasks that first emerge in adolescence. We created this special section to assemble studies that illustrate these principles in examining the traits, contexts, and experiences that underlie both neural and behavioral development.

Longitudinal studies serve as the gold standard in developmental science because they allow us to unravel dimensions of change rather than snapshots

(C) 2018 Society for Research in Child Development All rights reserved. 0009-3920/2018/8903-0001 DOI: $10.1111 /$ cdev. 13073 
in development. Thus, we showcase in this section studies that have data on brain development with at least two time points on the same individuals (e.g., longitudinal within-person designs; pre-post training or treatment designs) and draw on a range of neuroimaging and electrophysiological methods (e.g., structural MRI, functional MRI [fMRI], electroencephalography [EEG]). Two sets of articles provide evidence for delineating windows of brain development based on within-person changes in the brain's structure or function in relation to a range of domains of functioning. The first set of articles focus on brain development in support of early emerging functions in infancy and childhood. The second set of articles focus on brain change, growth, and reorganization during adolescence that foster maturation toward adult-like behaviors (e.g., role of the frontal lobes in cognitive control; identity development). This type of evidence is critical to understanding how measures of brain function and structure can inform child and adolescent development.

\section{We Need to Examine Within-Person Change in the Brain}

Brain development reflects time- and experiencedependent dynamic processes of growing, creating new connections, or advancing functioning as well as loss processes such as pruning, closing of critical or sensitive periods, or regressing in functioning. In developmental neuroscience, cross-sectional designs have been frequently used to generate knowledge about the degree to which specific neural characteristics and processes are present within certain age groups compared to each other (e.g., 9-12 year olds vs. 13-17 year olds vs. 18-25 year olds; 6-9 months vs. 12-15 months). Based on such designs, striking mean-level differences exist between age groups in specific domains. For example, reward cues, salient emotions, and peer influence are found to elicit heighted brain activity in adolescence relative to childhood or adulthood (Chein, Albert, O'Brien, Uckert, \& Steinberg, 2011; Galvan et al., 2006; Guyer et al., 2008). In addition, we have documented how the size of, and connections among, brain structures change over time drawing on age cohorts (e.g., prefrontal cortex [PFC]; Giedd et al., 2015).

We know less about how within-person change unfolds in the process of brain growth and advancement in function. For example, by examining slopes, we can gain knowledge about the timing and progression or regression of brain development such as when deviances from expected patterns occur as well as patterns of tempo, stability, and change. This is information we cannot gain from comparing age-categorized groups on mean levels of a developmental outcome. Longitudinal designs are also important for understanding how developmental changes in the brain co-occur with changes in behavior, relations that are unobservable with cross-sectional data. Indeed, gathering longitudinal data will enable our ability to tie neural changes directly to changes in our constructs of interests, and expand our understanding of both normative mechanisms and markers of individual variability and change.

Grimm, Davoudzadeh, and Ram (2017) highlight how nearly 40 years ago, Baltes and Nesselroade (1979) offered five motivations for conducting longitudinal research. These reasons included (a) identifying intraindividual change/stability, (b) identifying interindividual differences/similarity in intraindividual change, (c) analyzing interrelations in change in multiple constructs, (d) analyzing causes/determinants of intraindividual change, and (e) analyzing causes/determinants of interindividual differences in intraindividual change. These rationales encourage us to do the same in research on brain development and individual trajectories across neural systems, as they are necessary for moving beyond cross-sectional snapshots of brain maturation.

Indeed, recent perspectives have highlighted the benefits of using within-person longitudinal designs to study brain development. For example, longitudinal designs can reduce sampling bias introduced by cohort effects, help tease apart trait stability from state variability in brain and behavior, demonstrate brain-context interactions, and be used for testing mediation models of causal factors in development (Crone \& Elzinga, 2015). Fortunately, collecting longitudinal measurements of the brain has gained traction in ways that are advancing the field by shedding light on the degree of stability, concordance between brain and behavior, and which stimuli and environmental inputs are more or less influential for different brain regions in commonly assessed brain metrics such as regional volumes or neural reactivity.

\section{Examples of Opportunities for Neurodevelopmental Plasticity}

As discussed next, the 11 studies in this special section represent a wide-ranging set of brain-based 
functions and characteristics including (a) inputs that predict specific brain indices over time or elicit variation in brain reactivity, (b) brain connectivity at rest without specific inputs, and (c) individual differences related to features of long-term brain structure maturation. To assess brain reactivity and connectivity, the studies presented in this section used EEG (Lahat et al., 2018; MacNeill, Ram, Bell, Fox, \& Pérez-Edgar, 2018) and event-related potentials (ERPs; Brooker, 2018; Pickron, Iyer, Fava, \& Scott, 2017) in early development, with the exception of Lahat et al. who examined adolescents. To study change in adolescence and early adulthood, five studies used either fMRI while participants were at rest (Sylvester et al., 2017) or when paired with a task (Lauharatanahirun et al., 2018; Qu, Pomerantz, McCormick, \& Telzer, 2018; Schreuders et al., 2018; Vilgis et al., 2018). Finally, two studies assessed brain structure using MRI to quantify the volume of specific brain regions or the thickness and surface of cortical areas (Becht et al., 2018; Ferschmann et al., 2018).

\section{Infancy and Early Childhood}

Infancy and early childhood mark a significant period for relatively rapid brain growth. The brain reaches nearly adult size by the age of 5 , initial connections are laid down, and a multitude of experience-dependent changes support emerging individual differences in functioning (Giedd \& Rapoport, 2010; Lenroot \& Giedd, 2006). Indeed, these brain changes support fundamental processes such as language acquisition, attention, working memory, and self-regulation (Johnson, 2000, 2001, 2011). Three studies in this special section focus on within-person change in brain activity during infancy and early childhood. Pickron et al. (2017) conducted an innovative intervention study over 3 months to examine change in infant brain response using ERPs during a visual attention task. The study randomly assigned 43 six-month-olds to either a parent-delivered training group or to a notraining group. Brain activity was measured before and after the 3-month long training, with a focus on the Nc component of ERP, an index of selective and sustained attention. Infants trained to individuate labeled object exemplars increased their attention to these novel objects manifested as a larger Nc amplitude to infrequently presented objects. This suggests that a top-down environmental influence, here label-training, altered a specific neural substrate of attention during this period of development. Thus, from 6- to 9-months of age, the infant brain showed plasticity in response to repeated previous learning when attending to novel objects.

A study by MacNeill et al. (2018) examined whether changes in brain electrical activity measured using EEG paralleled the timing (how mature infants are relative to same-age infants) and rate (how quickly or slowly infants gained proficiency) of change in object permanence performance. A sample of 28 six-month-olds was tested monthly for 7 months on the classic A-not-B task and electrocortical brain activity was assessed at each visit. A nonlinear pattern of performance was found with most infants unsuccessful on the task at 6 months, a rapid nonlinear increase in performance from 7 to 11 months, and peak performance at ceiling by 12 months. Baseline EEG power increased linearly from 6 to 12 months of age across the brain. Importantly, infants who advanced more quickly in task performance had lower occipital (but not frontal) EEG at age 6 months and showed relatively small increases in occipital EEG power over time. The coupling between EEG and performance suggests developmental plasticity specifically of occipital neural systems involved in attending to and tracking moving objects supported the increased ability to attend to visual cues and facilitated learning during the first year of life. MacNeill et al.'s results also highlight the importance of modeling both linear and nonlinear change patterns in early brain and behavioral development and charting their progression at behavior-relevant time intervals, two goals met through repeated measurement of brain and behavior within-individual during the window of skill acquisition.

The third article covering this period of development focused on an aspect of self-regulation that begins to mature in the preschool years. Using a 1-year prospective longitudinal design, Brooker (2018) examined in 119 three-year-olds a neural substrate of self-monitoring indexed by error-related negativity (ERN) via EEG recordings of electrical brain activity. ERN is thought to be an adaptive mechanism signaling the need for cognitive resources for self-control. Developmental plasticity of the brain was investigated in relation to the sensitivity of parents to their child's behaviors and a more distal environmental factor marked by socioeconomic status (SES). Normative developmental increases in ERN amplitude from age 3 to 4 were found for preschoolers raised in families with high maternal sensitivity and high SES. Results suggest that this neural system implicated in self-monitoring may become organized earlier in development for children raised in more enriched and responsive environments. 
As a whole, these articles highlight that the infant brain changes over time in response to specialized learning experiences and that the timing and rate of change in specific areas of the brain parallel increased cognitive proficiency within a specific window of time. Furthermore, normative development of a brain system that contributes to self-regulation is supported by optimal environmental input over the course of a year across early childhood. These studies are innovative examples of examining brain activity in young children because they open a new focus on very early appearing neurally based change processes that underlie developmentally appropriate domains of functioning and account for the influence of input from early caregivers in facilitating these key developmental functions via brain changes.

\section{Adolescence and Early Adulthood}

We have also come to learn that adolescence represents another rapid and dynamic growth period during which the brain changes in critical ways that support more nuanced, complex and controlled behaviors such as decision making, reward sensitivity, emotion regulation, and making sense of complex social experiences (Casey, 2015; Guyer, Silk, \& Nelson, 2016; Nelson, Jarcho, \& Guyer, 2016; Steinberg, 2010). Adolescent brain refinement occurs through changes in concentrations of gray and white matter, reinforcement of short- and longrange neural connections, linear and nonlinear changes in brain reactivity, and modulation in levels of specific neurotransmitters, such as dopamine (Spear, 2013). In addition, we now understand that many of these changes extend well into early adulthood, lengthening the traditional time window of neural development and mirroring the modern day lengthening of the transition period into adulthood, referred to as emerging adulthood (Arnett, 2007; Taber-Thomas \& Perez-Edgar, 2015).

EEG and MRI have been the two most widely used tools for studying the adolescent brain in vivo. MRI has been useful because it provides high spatial resolution of brain structures and allows statistical maps representing neural activity to be aligned with anatomy. At about 7-9 years of age, children are more capable of laying very still in the scanner (important for obtaining clear pictures of the brain) and can more easily follow what can be relatively complex rules required to complete tasks paired with fMRI or EEG. Six articles in the special section used fMRI or EEG to study changes in adolescent brain activity either at rest or in response to a task.
Measuring brain activity at rest can provide information regarding variations in functional networks that are intrinsic to the individual and independent of specific tasks. Variations in intrinsic connectivity can help explain how the individual's brain responded to past experience and set the foundation for predicting future responses. Two articles in the special section assessed neural connectivity at rest in order to understand the associations between shyness, evolving brain profiles at rest, and socioemotional development.

Sylvester et al. (2017) examined whether shyness predicted change in resting state neural connectivity across three time points in middle childhood and early adolescence in 147 youth. Temperamental shyness is an early appearing individual trait that is relatively stable over time and, when coupled with specific additional risk factors, can lead to a marked increase in risk for anxiety (Pérez-Edgar \& Guyer, 2014). Over the course of development, changes in neural connectivity are thought to underlie more effective and efficient regulatory mechanisms, potentially blunting the risk for developing psychopathology. In this study, the authors targeted four functional brain networks associated with both temperamental shyness and anxiety: default mode $(\mathrm{DMN})$, frontoparietal, salience, and ventral attention. Of the four networks, they found that only the trajectory of functional connectivity in the DMN was associated with shyness, even after controlling for anxiety symptoms. In particular, participants with low levels of shyness, and therefore low risk, presented a negative slope in connectivity over time. In contrast, highly shy children were relatively flat in their levels of DMN connectivity over time. The resulting relative hyperconnectivity from late childhood to early adolescence could support a shift in a more internally focused and self-referential type of thinking, characteristics previously highlighted as symptomatic of anxiety. In this way, early temperament is embedded in specific brain networks, in this case one that involves self-focus highlighting a new cognitive-behavioral target to probe in interventions for children at risk for anxiety due to their early life temperament.

Lahat et al. (2018) also focused on shyness, this time examining the construct in the context of maltreatment in order to predict change in resting EEG asymmetry. Here, the focus was on 80 adolescent girls (ages 14-16) who had previously experienced child maltreatment. Although maltreatment often has devastating effects, it is difficult to predict the outcomes for any one child. Examining biomarkers such as brain activity may boost prediction and 
point to targets of intervention and opportunities to leverage brain plasticity. Resting EEG asymmetry, in turn, is a low-cost, easily tolerable, marker of electrocortical activity. Left frontal EEG activity at rest, and in result to provocation, is associated with approach-related behaviors and emotions. In contrast, right frontal EEG activity is associated with withdrawal-related behaviors and emotions, early temperamental shyness, and the later emergence of anxiety and depression. Lahat et al. (2018) found that adolescent girls exposed to child maltreatment reported higher levels of shyness than control participants did only if they also presented with greater relative right frontal EEG asymmetry. In addition, adolescents exhibiting right frontal EEG asymmetry at rest had both the highest and lowest shyness scores, depending on their maltreatment history. Adolescent girls with left frontal EEG asymmetry at rest appeared relatively less sensitive to their environment. This finding is in line with the differential susceptibility to the environment model (Ellis, Boyce, Belsky, Bakermans-Kranenburg, \& van Ijzendoorn, 2011), suggesting that right frontal EEG asymmetry may make children more open to environmental input, for better and for worse including during the middle adolescent period beyond the early childhood period emphasized in the susceptibility model.

Four articles used task-based fMRI to elicit neural response to specific cues or images, which allows researchers to assess the regions of the brain that are more or less reactive to specific classes of stimuli. Adolescence marks a time of increased risk for depression, especially in girls (Avenevoli, Swendsen, He, Burstein, \& Merikangas, 2015). This risk may relate to the cognitive approaches girls use to manage their emotions. Vilgis et al. (2018) undertook an innovative analytic approach using a cross-lagged panel analysis to test the influence of brain and behavior in the context of risk for depression in adolescent girls. Using fMRI, brain reactivity was measured in a diverse sample of 78 sixteenyear-old girls (nearly two thirds were AfricanAmerican) when they appraised their subjective feelings of sadness in response to images of others depicting sad facial expressions. Signs of depression and the strategies the girls use to regulate their emotions were also assessed. The girls were reevaluated at age 17 for depression, emotion regulation, and brain reactivity to sad faces. The study found that the level of brain activity associated with reflecting on one's sadness at age 16 in the dorsomedial PFC served as a predictor of depression severity and the ability to effectively regulate emotions at age 17. In addition, this neural sensitivity during sadness introspection predicted specific strategies, which fed variation in regulatory effectiveness. Using a cross-lagged panel analysis in this way allowed for the determination of whether specific patterns of neural sensitivity to thinking about one's emotions predicts behaviors (e.g., emotion strategy usage) or vice versa. These findings suggest that brain activity may drive emotional behaviors across this sensitive developmental period for depression risk, which in turn can inform targets of intervention (e.g., thought processes, emotion probes) during this late adolescent window that may alter emotion regulation via the brain for girls at heightened risk for depression.

In addition to spikes in anxiety and depression, adolescence brings a normative increase in risk taking and reward seeking behaviors (Romer, Reyna, \& Satterthwaite, 2017; Steinberg, 2008). Risk taking can facilitate the development of skills needed to go out into the world as an independent and autonomous individual, while also providing experiences that scaffold adult roles. Three studies in this special section examined how the brain changes over time in relation to risk taking and reward processing.

Qu et al. (2018) examined whether the neurodevelopment of cognitive control associated with changes in the PFC across adolescence would be altered as a function of adolescents' perceptions of teens as being irresponsible. The authors hypothesized that youth who view the teenage years as marked by irresponsibility and lack of familial connection would lag in the development of cognitive control and engage in increased risk taking. A three-wave longitudinal neuroimaging study of twenty-two 13-year-olds examined the youths' stereotypes about teens. Middle schoolers who reported that teens are irresponsible in the family showed increased bilateral ventrolateral PFC (vlPFC) activation during a cognitive control task across the transition to high school. Although vlPFC activity is typically associated with impulse control, interindividual declines in vlPFC over time may reflect more mature, and efficient, neural control. Indeed, both stereotypes of teen irresponsibility and increased vlPFC activation over time were associated with increased risk taking behaviors. These findings provide preliminary evidence that youth's conceptions of adolescence play a role in neural plasticity over this phase of development, perhaps by coloring their experience with, and interpretations of, their families. That the processing of these perceptions permeates the level of the brain 
suggests that taking on new mindsets and ways of thinking about others are quite powerful and can serve as targets for social cognitive interventions at this phase of development.

Lauharatanahirun et al. (2018) also examined adolescent risky behavior. However, here the focus was on whether risky behaviors were related to developmental changes in decision making and associated neural correlates as a function of the family environment. In this study, 167 adolescents (13-15 years, 53\% male) self-reported on the level of chaos they experienced in their homes and their parents' monitoring practices. Participants then completed a decision-making task during fMRI at two time points 1 year apart. The insular cortex has been associated with processing risk information and increased activity can lead to risk aversion (Mohr, Biele, \& Heekeren, 2010). Parental knowledge of their adolescent's whereabouts and behaviors was positively related to insular activity at both time points, suggesting that adolescents' perception of parental monitoring impacted their consideration of risk. However, this effect only held among adolescents in low-chaos environments. It also appears that the consistency of input is an important consideration when understanding the role the environment may play in shaping the neural response to developmentally salient processes such as deliberating whether to engage in risky behaviors. This is particularly central when the focus is on the adolescent's subjective assessment of his or her environment.

Finally, Schreuders et al. (2018) examined how ventral striatum responses to rewards develop across adolescence and early adulthood. They brought a specific focus on the relation between changes in striatal response and individual differences in state- and trait-level reward sensitivity. Participants (aged 8-29 years) were tested across three waves separated by 2 years (693 fMRI-scans in total) in an accelerated longitudinal design. Previous work suggests that striatal activity is closely associated with adolescents' tendency to seek out rewards and engage in exciting (albeit risky) behaviors. This large-scale study confirms the adolescent peak in reward-related ventral striatum, specifically in the nucleus accumbens (NAcc), activity. In addition, the rise in striatal activity over time was strongest among individuals with greater trait-level reward sensitivity in the younger half of the sample, leading up to mid-adolescence. This relation was not evident in the older half of the sample, who was on the downward slope for both striatal reactivity and reward sensitivity. This study demonstrates that state- and trait-level reward sensitivity differentially account for reward-related ventral striatum activity in different phases of adolescence and early adulthood. Specifically, the expectation that ventral striatum activity peaks in mid-adolescence and declines again in late adolescence and beyond, especially for youth driven to seek novel experiences was confirmed. The data suggest narrow developmental windows within the adolescent period may be designed to foster youths' exploration of new experiences and increase the chance for new environmental influences to occur.

How much of our brain development is explained by our attributes and approach to the world as individuals? Using structural MRI, Ferschmann et al. (2018) examined how personality traits relate to brain development in anatomical characteristics, an important but understudied question. These researchers assessed cortical thickness (CT) and surface area (SA) in 99 participants aged 8-19 years at an initial time point and again 2.6 years later. Specific personality traits from the Big Five Model of Personality were related to longitudinal change in regional CT or SA. In particular, conscientiousness, emotional stability, and imagination were associated with greater age-expected cortical thinning over time. However, few age-independent cross-sectional associations were found between personality traits and CT or SA. Together, these results suggest that the timing and pattern of change may be more informative when examining individuals still in the formative processes of personality development. Static brain-behavior relations may be more evident, and likely more stable, in adults who show relatively less change at both the neural and behavioral level of analysis, but longer term longitudinal studies are needed to see whether this pattern persists across the life span. These data could help us better understand variations in personality and emotional behavior evident in life span research extending from young adulthood into late adulthood.

Identity formation is a fundamental component of adolescent development (Meeus, 2011). Becht et al. (2018) conducted a multimethod multisample longitudinal study to investigate how the volume of brain structures associated with self-reported goal-directedness and information-seeking relate to adolescents' global identity. Their focus was on how perceptions of interpersonal and educational functioning influence their identity. The first study (497 adolescents; $M_{\text {age }}$ T1 13.03 years) collected selfreport data on goal-directedness across three biannual waves with identity measured one wave 
later. Study 1 design and measurements were repeated in Study 2 (131 adolescents; $M_{\text {age }}$ T1 14.69 years) and extended with structural gray matter volume data for the NAcc and PFC, collected across three biannual waves. They found relative stability for the NAcc and decreases in PFC volume over time. Using latent growth curve models, they found that NAcc volume was most strongly associated with identity formation, which might reflect striatal involvement in novel and rewarding behavior. In contrast, PFC volume predicted identity maintenance, perhaps reflecting prefrontal roles in cognitive control and behavior monitoring. Importantly, these individual differences were noted with measures that showed relative (rank-order) stability. It is only in examining trajectories that many subtle relations are able to emerge. These findings demonstrate how having a strong sense of identity was shaped by behaviors, pursuing one's goals, and in an area of the brain that supports motivated action and experiencing rewards, highlighting the concordance between brain and behavior in developing one's self-concept.

\section{Future Directions}

The current special section set out to highlight diverse approaches to a common set of questions: How do we capture change in the brain and can we then link change to observed patterns of thought and behavior? Importantly, we situated these questions within two unique developmental periods that manifest significant modeling of the brain through progressive and regressive processes. The articles presented here vary in a number of features, including the age of participants, the core technology used, the psychological construct of interest, and the approach to quantifying the interrelated systems associated with developmental change. This diversity speaks to the exciting road ahead for future research studies building on work to date.

First, we need longitudinal studies starting just prior to key transition or turning points in development to determine benchmarks of neurodevelopment in structure and function. Benchmarks will then aid in formulating underlying principles of brain development based on the timing and rates of growth/maturation as suggested, for example, by the findings of MacNeill et al. (2018) showing that A-not-B neural correlates parallel behavioral competency that then plateaus. While some work has begun to formulate indices of brain maturation and brain development phase (Brown et al., 2012; Cao et al., 2015), including evidence that brain-derived age correlates well with chronological age (Bunge \& Whitaker, 2012), we currently do not have anything close to the weight and height charts used to measure physical development. We do not have population-based metrics, such as the T-scores we often use to characterize socioemotional behavior. Thus, our conclusions regarding normative development, comparisons between typical and atypical functions, or brain associations with maladaptive behavior are all dependent on the specific characteristics of the single study we are assessing and the comparison sample chosen by the researchers. Given that most of the current literature depends on small samples dominated by convenience samples, we have little sense of how one set of data compare with to-be-expected patterns of structure, function, and development.

There are now large-scale studies (e.g., ABCD, IMAGEN, Developing Human Connectome Project, Pediatric Imaging, Neurocognition, and Genetics Project) that may help fill in the missing gaps. Rosenberg, Casey, and Holmes (2018) recently summarized existing large MRI cohort studies ( $>500$ participants) and showed that most of these studies are conducted in the United States and Western Europe, but several new cohort studies are now also ongoing in South America and China. These new developments highlight continued progress in understanding brain development in individuals across the globe (Rosenberg et al., 2018). Centralized aggregation databases, such as the Neuroimaging Informatics Tools and Resources Clearinghouse, are also hugely beneficial to improving our power to detect change and growth in the brain. However, just as we are carefully charting the length and width of brain development, we must also focus on capturing how variations in brain development link to accompanying variation in behavioral development.

For example, one avenue for research will likely focus on understanding how the epigenetics of biological traits (such as temperament) set the foundation for brain development by both constraining the form of specific neural structures and functions and influence the impact of the genetic and environmental input that fuel experience-expectant and experience-dependent changes. That is, certain biological traits may lead individuals to seek out environments that further reinforce nascent developmental tendencies propagating modification of gene expression through epigenetics. Thus, variations in sensitivity to environmental input and the input 
provided by the environment may not be fully orthogonal. Further complicating the brain-to-environment-to-brain dynamic is the fact that the daily determinants of environmental input will also change with development, which aligns with the perspective that epigenetic models are more applicable to brain development than heritability models (see Lester, Conradt, \& Marsit, 2016).

For the young child, primary caregivers, including siblings, extended family members, and others who fulfill the child's needs, shape, control, and filter the environment. Thus, the personal and psychosocial characteristics of the child's main caregivers influence how the caregiver interacts with the child. Caregiver behaviors, in turn, shape the child's experienced environment. In this way, caregiver behaviors and personality may affect how infants come to view and approach the world.

Further engagement with the world, in turn, shapes brain mechanisms that are uniquely plastic, ready to learn from experience and adapt to the environment (Selemon, 2013). Continuing this chain, the brain changes that emerge via plasticity mechanisms in turn influence the environments that individuals seek out as children transition from childhood to adolescence and young adulthood, taking greater control of their daily experiences. We will need nimble technologies embedded in rich empirical research protocols applied across time and context in order to capture this rich, and dynamic, process.

Of course, the interval of brain assessment must be titrated to the change seen in targeted behaviors-for infants, change can occur within days, week and months. As such, observation intervals may need to be frequent and tightly scheduled in order not to miss the window of change. Our methods also need to be sensitive to the infant's ability (or willingness) to tolerate our measures. Finally, we will need to step beyond traditional approaches that have limited infant research to small samples with limited measures per child (see Oakes, 2017, for a critique).

As we move through development, we open up the developmental window. For example, the changes we anticipate seeing in adolescence may be more gradual, as some behaviors emerge over the course of years. When we slow the pace of development, we often introduce greater variability, as the system has more opportunity to absorb and respond to variations in the environment and experience. Thus, the measures we are most interested in for older children (e.g., social competence) are often complex, subtle, and difficult to quantify.
Here, the goal will be to find procedures that are amenable to our measures of the brain, such as fMRI, and the corresponding metrics of the brain, without sacrificing the ecological validity of our constructs of interest.

Given the time and resource intensive nature of longitudinal studies, trade-offs often need to be made. As seen in the current special section, and in the larger literature, researchers either focus on relatively smaller samples but institute rich and multilayered observation of development, or they take a population approach, with less intensive measures that allow for more heterogeneous and variable samples. Longitudinal studies, by definition, are designed to capture a phenomenon over time. As such, they fix at study outset the methodologies and measures that often must be carried out without change within and across time. In vivo brain measures that are well tolerated by children across age are an asset in this regard. Shared measures allow for direct comparisons across the entire cohort at any one wave of study (T1) and over the life of the study (T1 vs. T2 vs. T3, etc.).

Even though longitudinal studies help up to understand co-varying changes over time, they do not allow us to draw conclusions about causality. For example, correlated changes in brain growth (e.g., PFC development) and behavioral measures (e.g., alcohol use) may be related to a third variable (e.g., temperament). Nonetheless, longitudinal change studies are more sensitive in detecting rapid periods of growth, which can, for example, inform when a child is more sensitive to environmental input. Often short-term experimental studies can help probe the specific mechanisms that fuel patterns and relations evident in relatively slow-moving longitudinal studies. For example, cognitive enrichment or training studies can help delineate how environmental factors accelerate or delay brain development. Importantly, experimental methods can systematically manipulate processes or mechanisms that are thought to directly shape outcomes of interest. Coupled with in vivo brain imaging techniques, we need to isolate agents of change as well as the specific targets of change. These studies will directly impact translational work aimed at creating effective interventions that may help re-direct maladaptive trajectories.

Additionally, the field needs to systematically study the culture and context in which our constructs of interests, and agents of neurodevelopmental change, are embedded (Chiao, 2017). That is, much of the literature to date has taken a relatively Universalist view, working under the 
assumption that the developmental trajectories, underlying mechanisms, and environmental processes identified can be equally applied across culture and context. Despite the recent calls for broadening developmental research to include nonWestern populations, the field is biased toward WEIRD: Western, educated, industrialized, rich, and democratic populations (Henrich, Heine, \& Norenzayan, 2010; Legare \& Harris, 2016). This limitation is evident in the articles included in the current special section. The bias not only encompasses who is being studied, but also reflects who is doing the studying.

Developmental neuroscience has progressed by working to incorporate the role of context and environmental input on brain-behavior relations. The next step is to systematically study a broader array of contexts and cultures to note not only similarities or differences across groups, but to better understand the mechanisms that help shape these differences. Until we do so, we cannot fully understand the breadth and scope of our phenomena of interest. To borrow from the world of motor development, the standard consensus regarding the timing and progression of motor development is upended when you consider children developing in diverse contexts that both limit (e.g., bundling) and expand (e.g., early tool use) the ability to engage in behavior. If we wish to chart the extent of brain plasticity in development, we must incorporate the breadth of experiences and contexts a child may be embedded in.

We hope that this special section on the developing brain will inspire and inform future work that focuses on developmental processes and mechanisms involving the temporality of change, such as (a) identifying neural regions with differing maturational timetables and quantifying the impact of their maturation on developmental outcomes; (b) examining sensitive periods in brain development rooted in cognitive or behavioral processes using experimental interventions informed by nonlinear developmental patterns that give clues regarding inflection points for sensitive periods; (c) elucidating individual differences in rates of brain maturation and their developmental consequences; and (d) considering how early alterations in the brain affect later brain development.

\section{References}

Arnett, J. J. (2007). Emerging adulthood: What is it, and what is it good for? Child Development Perspectives, 1, 68-73. https: / / doi.org/10.1111/j.1750-8606.2007.00016.x
Avenevoli, S., Swendsen, J., He, J. P., Burstein, M., \& Merikangas, K. R. (2015). Major depression in the National Comorbidity Survey-Adolescent supplement: Prevalence, correlates, and treatment. Journal of the American Academy of Child and Adolescent Psychiatry, 54, 37-44. https://doi.org/10.1016/j.jaac.2014.10.010

Baltes, P. B., \& Nesselroade, J. R. (1979). History and rationale of longitudinal research. In J. R. Nesselroade \& P. B. Baltes (Eds.), Longitudinal research in the study of behavior and development (pp. 1-39). New York, NY: Academic Press.

Becht, A. I., Bos, M. G. N., Nelemans, S. A., Peters, S., Vollebergh, W. A. M., Branje, S. J. T., . . Crone, E. A. (2018). Goal-directed correlates and neurobiological underpinnings of adolescent identity: A multimethod multisample longitudinal approach. Child Development. https: / / doi.org/10.1111/cdev.13048

Brooker, R. (2018). Maternal behavior and socioeconomic status predict longitudinal changes in error-related negativity in preschoolers. Child Development. https://doi. org/10.1111/cdev.13066

Brown, T. T., Kuperman, J. M., Chung, Y., Erhart, M., McCabe, C., Hagler, D. J., . . . Dale, A. M. (2012). Neuroanatomical assessment of biological maturity. Current Biology, 22, 1693-1698. https://doi.org/10.1016/J.CUB. 2012.07.002

Bunge, S. A., \& Whitaker, K. J. (2012). Brain imaging: Your brain scan doesn't lie about your age. Current Biology, 22, R800-R801. https://doi.org/10.1016/J.CUB. 2012.07.032

Cao, B., Mwangi, B., Hasan, K. M., Selvaraj, S., Zeni, C. P., Zunta-Soares, G. B., \& Soares, J. C. (2015). Development and validation of a brain maturation index using longitudinal neuroanatomical scans. Neurolmage, 117, 311318. https: / / doi.org/10.1016/j.neuroimage.2015.05.071

Casey, B. J. (2015). Beyond simple models of self-control to circuit-based accounts of adolescent behavior. Annual Review of Psychology, 66, 295-319. https://doi.org/10. 1146/annurev-psych-010814-015156

Chein, J., Albert, D., O’Brien, L., Uckert, K., \& Steinberg, L. (2011). Peers increase adolescent risk taking by enhancing activity in the brain's reward circuitry. Developmental Science, 14, F1-F10. https://doi.org/10. $1111 / j .1467-7687.2010 .01035 . x$

Chiao, J. Y. (2017). Cultural neuroscience of the developing brain in adolescence. In N. Budwig, E. Turiel, \& P. D. Zelazo (Eds.), New perspectives on human development (pp. 330-350). Cambridge, UK: Cambridge University Press. https://doi.org/10.1017/cb o9781316282755.018

Crone, E. A., \& Elzinga, B. M. (2015). Changing brains: How longitudinal functional magnetic resonance imaging studies can inform us about cognitive and socialaffective growth trajectories. Wiley Interdisciplinary Reviews: Cognitive Science, 6, 53-63. https://doi.org/10. 1002 /wcs.1327

Ellis, B. J., Boyce, W. T., Belsky, J., Bakermans-Kranenburg, M. J., \& van Ijzendoorn, M. H. (2011). Differential 
susceptibility to the environment: An evolutionary-neurodevelopmental theory. Development and Psychopathology, 23, 7-28. https://doi.org/10.1017/S095457941000 0611

Ferschmann, L., Fjell, A. M., Vollrath, M. E., Grydeland, H., Walhovd, K. B., \& Tamnes, C. K. (2018). Personality traits are associated with cortical development across adolescence: A longitudinal structural MRI study. Child Development. https://doi.org/10.1111/cdev.13016

Galvan, A., Hare, T. A., Parra, C. E., Penn, J., Voss, H., Glover, G., \& Casey, B. J. (2006). Earlier development of the accumbens relative to orbitofrontal cortex might underlie risk-taking behavior in adolescents. Journal of Neuroscience, 26, 6885-6892. https://doi.org/10.1523/ JNEUROSCI.1062-06.2006

Giedd, J. N., \& Rapoport, J. L. (2010). Structural MRI of pediatric brain development: What have we learned and where are we going? Neuron, 67, 728-734. https:/ / doi.org/10.1016/j.neuron.2010.08.040

Giedd, J. N., Raznahan, A., Alexander-Bloch, A., Schmitt, E., Gogtay, N., \& Rapoport, J. L. (2015). Child Psychiatry Branch of the National Institute of Mental Health longitudinal structural magnetic resonance imaging study of human brain development. Neuropsychopharmacology, 40, 43-49. https://doi.org/10.1038/npp.2014. 236

Grimm, K. J., Davoudzadeh, P., \& Ram, N. (2017). Developmental methodology: IV. Developments in the analysis of longitudinal data. Monographs of the Society for Research in Child Development, 82(Serial No. 325), 46-66. https://doi.org/10.1111/mono.12298

Guyer, A. E., Monk, C. S., McClure-Tone, E. B., Nelson, E. E., Roberson-Nay, R., Adler, A. D., . . Ernst, M. (2008). A developmental examination of amygdala response to facial expressions. Journal of Cognitive Neuroscience, 20, 1565-1582. https://doi.org/10.1162/jocn. 2008.20114

Guyer, A. E., Silk, J. S., \& Nelson, E. E. (2016). The neurobiology of the emotional adolescent: From the inside out. Neuroscience and Biobehavioral Reviews, 70, 74-85. https://doi.org/10.1016/j.neubiorev.2016.07. 037

Henrich, J., Heine, S. J., \& Norenzayan, A. (2010). The weirdest people in the world? Behavioral and Brain Sciences, 33, 61-83. https://doi.org/10.1017/ S0140525X0999152X

Johnson, M. H. (2000). Functional brain development in infants: Elements of an interactive specialization framework. Child Development, 71, 75-81. https://doi.org/10. $1111 / 1467-8624.00120$

Johnson, M. H. (2001). Functional brain development in humans. Nature Reviews Neuroscience, 2, 475-483. https:/ / doi.org/10.1038/35081509

Johnson, M. H. (2011). Interactive Specialization: A domain-general framework for human functional brain development? Developmental Cognitive Neuroscience, 1, 7-21. https: / /doi.org/10.1016/j.dcn.2010.07.003
Lahat, A., Tang, A., Tanaka, M., Van Lieshout, R. J., MacMillan, H. L., \& Schmidt, L. A. (2018). Longitudinal associations among child maltreatment, resting frontal electroencephalogram asymmetry, and adolescent shyness. Child Development. https://doi.org/10.1111/cdev. 13060

Lauharatanahirun, N., Maciejewski, D., Holmes, C., Deater-Deckard, K., Kim-Spoon, J., \& King-Casas, B. (2018). Neural correlates of risk processing among adolescents: Influences of parental monitoring and household chaos. Child Development. https://doi.org/ 10.1111/cdev.13036

Legare, C. H., \& Harris, P. L. (2016). The ontogeny of cultural learning. Child Development, 87, 633-642. https://doi.org/10.1111/cdev.12542

Lenroot, R. K., \& Giedd, J. N. (2006). Brain development in children and adolescents: Insights from anatomical magnetic resonance imaging. Neuroscience $\mathcal{E}$ Biobehavioral Reviews, 30, 718-729. https://doi.org/10.1016/j.ne ubiorev.2006.06.001

Lester, B. M., Conradt, E., \& Marsit, C. (2016). Introduction to the special section on epigenetics. Child Development, 87, 29-37. https:/ / doi.org/10.1111/cdev.12489

MacNeill, L. A., Ram, N., Bell, M. A., Fox, N. A., \& Pérez-Edgar, K. (2018). Trajectories of infants' biobehavioral development: Timing and rate of A-not-B performance gains and EEG maturation. Child Development. https: / / doi.org/10.1111/cdev.13022

Meeus, W. (2011). The study of adolescent identity formation 2000-2010: A review of longitudinal research. Journal of Research on Adolescence, 21, 75-94. https://doi. org/10.1111/j.1532-7795.2010.00716.x

Mohr, P. N. C., Biele, G., \& Heekeren, H. R. (2010). Neural processing of risk. Journal of Neuroscience, 30, 66136619. https://doi.org/10.1523/JNEUROSCI.0003-10. 2010

Nelson, E. E., Jarcho, J. M., \& Guyer, A. E. (2016). Social re-orientation and brain development: An expanded and updated view. Developmental Cognitive Neuroscience, 17, 118-127. https://doi.org/10.1016/j.dcn.2015.12.008

Oakes, L. M. (2017). Sample size, statistical power, and false conclusions in infant looking-time research. Infancy, 22, 436-469. https://doi.org/10.1111/infa. 12186

Pérez-Edgar, K., \& Guyer, A. E. (2014). Behavioral inhibition: Temperament or prodrome? Current Behavioral Neuroscience Reports, 1, 182-190. https://doi.org/10. 1007/s40473-014-0019-9

Pickron, C. B., Iyer, A., Fava, E., \& Scott, L. S. (2017). Learning to individuate: The specificity of labels differentially impacts infant visual attention. Child Development. https://doi.org/10.1111/cdev.13004

Qu, Y., Pomerantz, E. M., McCormick, E., \& Telzer, E. H. (2018). Youth's conceptions of adolescence predict longitudinal changes in prefrontal cortex activation and risk taking during adolescence. Child Development. https: / / doi.org/10.1111/cdev.13017 
Romer, D., Reyna, V. F., \& Satterthwaite, T. D. (2017). Beyond stereotypes of adolescent risk taking: Placing the adolescent brain in developmental context. Developmental Cognitive Neuroscience, 27, 19-34. https://doi. org/10.1016/J.DCN.2017.07.007

1Rosenberg, M. D., Casey, B. J., \& Holmes, A. J. (2018). Prediction complements explanation in understanding the developing brain. Nature Communications, 9, 589. https:/ / doi.org/10.1038/s41467-018-02887-9

Schreuders, E., Braams, B. R., Blankenstein, N. E., Peper, J. S., Güroğlu, B., \& Crone, E. A. (2018). Contributions of reward sensitivity to ventral striatum activity across adolescence and early adulthood. Child Development. https:/ / doi.org/10.1111/cdev.13056

Selemon, L. D. (2013). A role for synaptic plasticity in the adolescent development of executive function. Translational Psychiatry, 3, e238. https://doi.org/10.1038/tp. 2013.7

Spear, L. P. (2013). Adolescent neurodevelopment. Journal of Adolescent Health, 52, S7-S13. https://doi.org/10. 1016/j.jadohealth.2012.05.006
Steinberg, L. (2008). A social neuroscience perspective on adolescent risk-taking. Developmental Review, 28, 78-106. https:/ / doi.org/10.1016/j.dr.2007.08.002

Steinberg, L. (2010). A behavioral scientist looks at the science of adolescent brain development. Brain and Cognition, 72, 160-164. https://doi.org/10.1016/j.bandc.2009.11.003

Sylvester, C. M., Whalen, D. J., Belden, A. C., Sanchez, S. L., Luby, J. L., \& Barch, D. M. (2017). Shyness and trajectories of functional network connectivity over early adolescence. Child Development. https://doi.org/10.1111/cdev.13005

Taber-Thomas, B. C., \& Perez-Edgar, K. (2015). Brain development in emerging adulthood. In J. J. Arnett (Ed.), Oxford handbook of emerging adulthood (pp. 126141). Oxford, UK: Oxford University Press.

Vilgis, V., Gelardi, K. L., Helm, J. L., Forbes, E. E., Hipwell, A. E., Keenan, K., \& Guyer, A. E. (2018). Dorsomedial prefrontal activity to sadness predicts later emotion suppression and depression severity in adolescent girls. Child Development. https://doi.org/10.1111/ cdev.13023 